\title{
Photon mass and gravitational deflection
}

\author{
Antonio Accioly* and Ricardo Paszko \\ Instituto de Física Teórica, Universidade Estadual Paulista, Rua Pamplona 145, 01405-000 São Paulo, SP, Brazil
}

(Received 16 December 2003; published 17 May 2004)

\begin{abstract}
The deflection of a massive photon by an external gravitational field is energy-dependent. Interesting enough, any massive quantum particle, no matter what its spin is, undergoes dispersive deflection in external gravitational fields. Exploiting the dispersive deflection of the quantized massive electromagnetic radiation by the gravitational field of the Sun, we find an upper bound for the photon mass.
\end{abstract}

DOI: 10.1103/PhysRevD.69.107501

PACS number(s): 04.25.-g, 04.80.Cc

\section{INTRODUCTION}

The gravitational deflection of photons in the context of higher-derivative gravity (HDG) treated as a semiclassical model, i.e., in the approximation where the gravitational field is retained as a classical background while the matter field is quantized, is energy-dependent [1,2]. An interesting consequence of this result is that gravity's rainbows and HDG can coexist without conflict [3]. Based on the fact that the rainbow effect is currently undetectable, a new constraint on the value of the parameter related to the squared Ricci tensor term is found that improves the generally accepted theoretical bound [4] by a factor of $10^{14}$ [3]. How reliable is this result? The aforementioned constraint is of the same order as that obtained by testing the gravitational inverse-square law in the submillimeter regime [5]. The conception of a gravity model in which dispersive deflection may take place thus makes sense.

On the other hand, dispersive photon propagation does not occur within the context of semiclassical general relativity (GR) $[6-8]$. How can we reconcile these apparently conflicting results? In other words, why can photonic dispersive deflection take place in HDG but it cannot in GR? The response is directly related to the particle content of the two theories. Indeed, HDG, in addition to the usual massless spin-2 excitation of GR, possesses also massive spin-2 and spin-0 excitations [9]. The latter are responsible for the dispersive effects. Therefore, we expect that in the framework of GR, a massive particle, regardless of its spin, would undergo dispersive deflection, while a massless one would not. As we shall show in a future publication, this is indeed the case [10].

In this Brief Report, we restrict ourselves to the analysis of the energy-dependent deflection of a massive photon. Our motivation is to find an upper limit for the photon mass by exploiting the dispersive deflection of the quantized massive electromagnetic radiation by the Sun. Since the upper bound that we have obtained is comparable with the other existing bounds, it can be interpreted as a check of the validity of quantum field theory in the presence of external gravitational fields.

Natural units are used throughout. Our signature is $(+$, $-,-,-)$. The Riemann and Ricci tensors are defined, re-

*Electronic address: accioly@ift.unesp.br spectively, as $R_{\lambda \mu \nu}^{\rho}=-\partial_{\nu} \Gamma_{\lambda \mu}^{\rho}+\partial_{\mu} \Gamma_{\lambda \nu}^{\rho}-\Gamma_{\lambda \mu}^{\sigma} \Gamma^{\rho}{ }_{\sigma \nu}$ $+\Gamma_{\lambda \nu}^{\sigma} \Gamma_{\sigma \mu}^{\rho}$ and $R_{\mu \nu}=R_{\mu \nu \rho}^{\rho}$.

\section{DISPERSIVE GRAVITATIONAL DEFLECTION OF QUANTIZED MASSIVE ELECTROMAGNETIC RADIATION}

We shall analyze the propagation of the massive photon in a static gravitational field treated as an external field in two steps: finding the classical background and computing afterward the gravitational deflection angle of the photon in this background.

\section{A. Classical background}

As is well known, in general relativity the gravitational field is defined by the action

$$
S=\int \sqrt{-g}\left[\frac{2 R}{\kappa^{2}}-\mathcal{L}_{M}\right] d^{4} x,
$$

where $\kappa^{2}=32 \pi G$, with $G$ being Newton's constant and $\mathcal{L}_{M}$ is the Lagrangian density for the usual matter. In the linear approximation, i.e., $g_{\mu \nu}=\eta_{\mu \nu}+\kappa h_{\mu \nu}$, where $\eta_{\mu \nu}$ $=\operatorname{diag}(+1,-1,-1,-1)$, and in the de Donder gauge, the field equations related to the action at hand turn out to be

$$
\square \gamma_{\mu \nu}=-\frac{\kappa}{2} T_{\mu \nu},
$$

where $\gamma_{\mu \nu} \equiv h_{\mu \nu}-\frac{1}{2} \eta_{\mu \nu} h$ and $T_{\mu \nu}$ is the matter tensor which describes the physical system under consideration in special relativity. The general solution of the equations above for a point mass $M$ located at $\mathbf{r}=\mathbf{0}$ is

$$
h_{\mu \nu}(\mathbf{r})=\frac{M \kappa}{16 \pi r}\left(\eta_{\mu \nu}-2 \eta_{\mu 0} \eta_{\nu 0}\right) .
$$

The corresponding metric assumes the form

$$
g_{\mu \nu}=\eta_{\mu \nu}+\frac{2 M G}{r}\left(\eta_{\mu \nu}-2 \eta_{\mu 0} \eta_{\nu 0}\right),
$$

which is nothing but the linearized Schwarzschild metric. 


\section{B. Dispersive deflection}

For small fluctuations around the Minkowski metric $\eta$, the full metric can be written as

$$
g_{\mu \nu}(x)=\eta_{\mu \nu}+\kappa h_{\mu \nu}(x),
$$

where $h_{\mu \nu}(x)$ is the graviton field.

Consequently, the Feynman rule for the interaction of a massive photon with a static gravitational field treated as an external field is obtained from the action for the gravitational minimally coupled massive photon field,

$$
S=\int d^{4} x \sqrt{-g}\left[-\frac{B_{\mu \nu} B^{\mu \nu}}{4}+\frac{m^{2} W_{\mu} W^{\mu}}{2}\right],
$$

where $W^{\mu}$ is a vector field describing a spin-1 boson of mass $m$ and $B_{\mu \nu} \equiv \partial_{\nu} W_{\mu}-\partial_{\mu} W_{\nu}$, expanding around flat space using Eq. (2).

Accordingly, the vertex function for the massive-photon field-static-external-gravitational field is

$$
\begin{aligned}
V_{\mu \nu}\left(p, p^{\prime}\right)= & \frac{\kappa}{2} h^{\lambda \rho}(\mathbf{k})\left[-\eta_{\mu \nu} \eta_{\lambda \rho} p \cdot p^{\prime}+\eta_{\lambda \rho} p_{\nu} p_{\mu}^{\prime}\right. \\
& +2\left(\eta_{\mu \nu} p_{\lambda} p_{\rho}^{\prime}-\eta_{\nu \rho} p_{\lambda} p_{\mu}^{\prime}-\eta_{\mu \lambda} p_{\nu} p_{\rho}^{\prime}\right. \\
& \left.+\eta_{\mu \lambda} \eta_{\nu \rho} p \cdot p^{\prime}\right) \\
& \left.+m^{2}\left(-2 \eta_{\mu \lambda} \eta_{\nu \rho}+\eta_{\mu \nu} \eta_{\lambda \rho}\right)\right],
\end{aligned}
$$

where $p\left(p^{\prime}\right)$ denotes the momentum of the incoming (outgoing) massive photon. The momentum space gravitational field, $h_{\mu \nu}(\mathbf{k})$, is defined as

$$
h_{\mu \nu}(\mathbf{k}) \equiv \int d^{3} \mathbf{r} e^{-i \mathbf{k} \cdot \mathbf{r}} h_{\mu \nu}(\mathbf{r})
$$

From Eq. (1) we promptly obtain

$$
h_{\mu \nu}(\mathbf{k})=\frac{\kappa M}{4 \mathbf{k}^{2}} \eta_{\mu \nu}-\frac{\kappa M}{2} \frac{\eta_{\mu 0} \eta_{\nu 0}}{\mathbf{k}^{2}} .
$$

The unpolarized cross section for the scattering of the massive photon for the external gravitational field may be calculated from the expression

$$
\frac{d \sigma}{d \Omega}=\frac{1}{(4 \pi)^{2}} \frac{1}{3} \sum_{r=1}^{3} \sum_{r^{\prime}=1}^{3} \mathcal{M}_{r r^{\prime}}^{2}
$$

with $\mathcal{M}_{r r^{\prime}}=\epsilon_{r}^{\mu}(\mathbf{p}) \epsilon_{r^{\prime}}^{\nu}\left(\mathbf{p}^{\prime}\right) V_{\mu \nu}$, where $\epsilon_{r}^{\mu}(\mathbf{p})$ and $\epsilon_{r^{\prime}}^{\nu}\left(\mathbf{p}^{\prime}\right)$ are the polarization vectors for the initial and final vectorial bosons, respectively. Consequently, the cross section is given by

$$
\begin{aligned}
\frac{d \sigma}{d \Omega}= & \frac{2}{3} \frac{G^{2} M^{2}}{\mathbf{p}^{4}(1-\cos \theta)^{2}}\left(3 \mathbf{p}^{4}+\frac{3}{2} m^{4}+2 \mathbf{p}^{2} m^{2}\right. \\
& \left.+2 \mathbf{p}^{2}\left(\mathbf{p}^{2}+2 m^{2}\right) \cos \theta+\mathbf{p}^{4} \cos ^{2} \theta\right),
\end{aligned}
$$

where $\theta$ is the scattering angle.

Now, taking into account that $d \sigma / d \Omega=(r / \sin \theta)|d r / d \theta|$, we come to the conclusion that

$$
2\left(\frac{G M}{b}\right)^{2}=\frac{1-\cos \theta}{\left(2+\frac{m^{2}}{E^{2}-m^{2}}\right)^{2}+\frac{8}{3}\left(1+\frac{m^{2}}{E^{2}-m^{2}}\right)(1-\cos \theta) \ln (1-\cos \theta)-\frac{2}{3}(1-\cos \theta)^{2}}
$$

where $E$ is the energy of the incident photon and $b$ is the impact parameter.

For small angles, Eq. (3) reduces to

$$
\theta_{E}^{2}=\frac{\theta^{2}}{\left(1+\frac{m^{2}}{2\left(E^{2}-m^{2}\right)}\right)^{2}+\left(1+\frac{m^{2}}{E^{2}-m^{2}}\right) \frac{\theta^{2}}{3} \ln \frac{\theta^{2}}{2}}
$$

where $\theta_{E} \equiv 4 M G / b$ is the prediction of semiclassical general relativity for the deflection of photons of zero rest mass [6-8].

In the extreme relativistic limit, $E \gg m$, the preceding formula yields

$$
\theta_{\mathrm{sc}}=\theta_{E}\left(1+\frac{m^{2}}{2 E^{2}}\right)
$$

which is the prediction of semiclassical general relativity for the deflection of a massive photon in an external gravitational field. This result tells us that a beam of massive photons with different energies passing through an external gravitational field gives rise to a gravity's rainbow. It is interesting to note that this rainbow is made up of massive photons, i.e., it is a gravitational rainbow of massive particles.

If Eq. (4) is correct, it must reproduce in the nonrelativistic limit the prediction of classical general relativity for the deflection of a massive particle which is not bound to the Schwarzschild geometry. Performing the computation for $\mathbf{p}^{2} \ll m^{2}$, we get 
where $v$ is the speed of the incident particle, which is just the same result as that obtained in Einstein's geometrical theory [11-13].

\section{BOUNDING THE PHOTON MASS}

Examining Eq. (5) carefully, we see that the term $\theta_{E}\left(m^{2} / 2 E^{2}\right)$ is nothing but the correction to the prediction of semiclassical general relativity concerning the deflection of a massless photon [6-8] due to a finite photon mass. Therefore, an upper limit for the photon mass may be obtained from the expression

$$
m \leqslant 2 \pi \nu \sqrt{\frac{2 \Delta}{\theta_{E}}},
$$

where $\Delta$ is the difference between the measured deflection angle and the calculated deflection angle related to photons of zero rest mass and $\nu$ is the frequency.

The best measurements of the deflection of radio waves by the gravitational field of the Sun are at the level of 1.4 parts in $10^{4}$ [14], while the lowest frequency measurements used by radio astronomers are in the neighborhood of $2 \mathrm{GHz}$ [15], implying $m<10^{-40} \mathrm{~g}$. Of course, this upper limit is considerably weaker than the recent upper bound from Luo et al. [16] but comparable to other existing bounds [17], which, in a sense, is a major test of our tree-level calculations. On the other hand, the method we have devised is new and it exploits an interesting but otherwise unknown facet of semiclassical general relativity: dispersive propagation of massive particles in external gravitational fields.

\section{DISCUSSION}

The dispersive deflection we have found does not obey of course the equivalence principle. The basic reason for this violation of the equivalence principle at the tree level is the particle's mass: In our model, a massive particle, regardless of its spin, experiences dispersive deflection, whereas a massless one does not.

Nevertheless, despite the fact that our main concern here is the propagation of a massive photon on a classical background, a discussion of the current efforts regarding photon propagation within the phenomenology of quantum gravity [18] is certainly appropriate since quantum-gravity effects, which need not obey the equivalence principle, may exist [19].

Consider, in this vein, the new astrophysical text reported by Jacobson et al. [20], where synchroton radiation emitted by electrons circling in a magnetic field from the Crab nebula is analyzed. The observations of the radiation from this nebula place a stringent constraint on the likelihood of detecting the effects of quantum gravity on electrons. In other words, any modification of the dispersion relations of the electron that might be induced by quantum gravity should be ruled out. Fortunately, Ellis et al. [19] have shown that photons may evade this synchroton radiation constraint by violating the equivalence principle: There exists a linear modification of the dispersion relation for the photon, but not for the electron, which agrees with known constraints from the Crab nebula and elsewhere. The linear modification of the dispersion relation for the photon has its origin in a model of spacetime foam [21,22]. Accordingly, dispersive photon propagation is compatible with phenomenological quantum gravity.

To conclude, we call attention to the fact that in the semiclassical context, dispersive photon deflection can only take place if the photon is endowed with mass.

\section{ACKNOWLEDGMENTS}

A.A. thanks CNPq-Brazil and R.P. thanks CAPES-Brazil for financial support.
[1] A. Accioly, A. Azeredo, H. Mukai, and E. de Rey Neto, Prog. Theor. Phys. 104, 103 (2000).

[2] A. Accioly, H. Mukai, and H. Blas, Nuovo Cimento Soc. Ital. Fis., B 115, 1235 (2000).

[3] A. Accioly and H. Blas, Phys. Rev. D 64, 067701 (2001).

[4] J. Donoghue, Phys. Rev. Lett. 72, 2996 (1994).

[5] C. Hoyle et al., Phys. Rev. Lett. 86, 1418 (2001).

[6] D. Boccaletti, V. de Sabbata, C. Gualdi, and P. Fortini, Nuovo Cimento A 48, 58 (1967).

[7] A. Accioly, A. Azeredo, E. de Rey Neto, and H. Mukai, Braz. J. Phys. 28, 496 (1998).

[8] A. Accioly, S. Ragusa, E. de Rey Neto, and H. Mukai, Nuovo Cimento Soc. Ital. Fis., B 114, 595 (1999).

[9] I. Antoniadis and E. Tomboulis, Phys. Rev. D 33, 2756 (1986).

[10] A. Accioly and R. Paszko (unpublished).

[11] C. Misner, K. Thorne, and J. Wheeler, Gravitation (Freeman, San Francisco, 1973).

[12] A. Accioly and S. Ragusa, Class. Quantum Grav. 19, 5429
(2002).

[13] A. Accioly and S. Ragusa, Class. Quantum Grav. 20, 4963 (2003).

[14] C. Will, Living Rev. Relativ. 4, 4 (2001).

[15] D. Lebach et al., Phys. Rev. Lett. 75, 1439 (1995).

[16] J. Luo et al., Phys. Rev. Lett. 90, 081801 (2003).

[17] A. Goldhaber and N. Nieto, Rev. Mod. Phys. 43, 277 (1971).

[18] J. Ellis, N. Mavromatos, D. Nanopoulos, and A. Sakharov, astro-ph/0309144.

[19] J. Ellis, N. Mavromatos, and A.S. Sakharov, Astropart. Phys. 20, 669 (2004).

[20] T. Jacobson, S. Liberati, and D. Mattingly, Nature (London) 424, 1019 (2003).

[21] J. Ellis, N. Mavromatos, and D. Nanopoulos, Phys. Rev. D 61, 027503 (2000).

[22] J. Ellis, N. Mavromatos, and D. Nanopoulos, Phys. Rev. D 62, 084019 (2000). 